Revista Electrónica Complutense de Investigación en Educación Musical ISSNe: 1698-7454

http://dx.doi.org/10.5209/RECIEM.55090

\title{
Pintando sonidos a través de la Improvisación Colectiva en Educación Secundaria
}

\author{
Carlos Lage-Gómez ${ }^{1}$ y Roberto Cremades-Andreu²
}

Recibido: 17 de Mayo de 2017 / Aceptado: 30 de Octubre de 2017

Resumen. El presente artículo explora los procesos de aprendizaje en la improvisación colectiva a través de un estudio de Investigación Acción centrado en la musicalización de una serie de cuadros de una pintora profesional (de reconocido prestigio). El estudio se desarrolló en un Instituto de Enseñanza Secundaria de una localidad madrileña a lo largo de 14 sesiones con un grupo de $3^{\circ}$ de ESO (28 estudiantes), durante el tercer trimestre del curso 2010-2011, como un ciclo de un estudio de investigación. Las preguntas de investigación se agruparon en torno al análisis de los procesos de aprendizaje, aspectos emergentes, además de estimar la pertinencia de este procedimiento como una estrategia efectiva para utilizar en el aula de música en secundaria. Para la recogida de los datos se emplearon la observación participante y no participante, grabaciones audiovisuales, entrevistas y encuestas. En la discusión se plantea la naturaleza holística del aprendizaje determinada por la identidad del grupo, motivación, las emociones positivas, el ambiente y bienestar, enmarcada en el desarrollo de un proyecto didáctico transversal.

Palabras clave. Improvisación colectiva; investigación acción; educación secundaria; creatividades musicales; experiencia musical significativa.

\section{[en] Painting Sounds through Group Improvisation in Secondary Education}

\begin{abstract}
This article explores the learning processes in group improvisation through a collaborative action research study centred on the "musicalization" of a series of paintings by a professional painter. The study took place with a group of third year students attending a Secondary School in Madrid over the course of 14 lessons during the third term of the year 2011. The research questions are based on the learning process analysis, the emergent aspects, and the analysis of the relevancy of group improvisation in Secondary Education. For the data analysis we used participant and non-participant observation, video recordings, interviews and questionnaires. The conclusion reveals the holistic nature of the learning processes determined by group identity, motivation, positive emotions, class environment and wellbeing in a transversal didactic project.
\end{abstract}

Keywords. Group improvisation; action research; secondary education; musical creativities; musical significant experience.

Sumario. 1. Introducción. 2. Método. 3. Análisis y exposición de los datos. 4. Implicaciones para la práctica docente en Secundaria. 5. Discusión. 6. Referencias biblográficas.

Cómo citar: Lage, C. y Cremades-Andreu, R. (2018). Pintando sonidos a través de la Improvisación Colectiva en Educación Secundaria, 15, 61-82.

\footnotetext{
$1 \quad$ Universidad Complutense de Madrid

E-mail: calage@ucm.es

2 Universidad Complutense de Madrid

E-mail: rcremade@ucm.es
} 


\section{Introducción}

Elliott y Silverman (2015) proponen un acercamiento filosófico a la educación musical, considerando, al igual que Small (2005), que la música es una forma de conocimiento en sí misma donde se fomenta el pensamiento creativo (Webster, 2002) en la búsqueda de experiencias musicales significativas para el alumnado (Webster, 2002), proceso que se podría relacionar con la teoría de las inteligencias múltiples (Gardner, 2011). Desde esta perspectiva, la creatividad emerge como un elemento trascendental en el ámbito de la educación musical, como ponen de manifiesto las propuestas didácticas pioneras de Schafer, Paynter, Self, Reibel o Gagnard, entre otros, en los años sesenta y setenta del siglo XX (Jorquera, 2010). Su reflejo en el currículo educativo español queda recogido en la Ley de Ordenación General del Sistema Educativo (LOGSE, 1990), con la que se abre la puerta de forma explicita al desarrollo de la creatividad en el currículo de Música en secundaria a través de la creación musical. Y es que una parte sustancial de la investigación sobre creatividad en educación musical en los últimos años (Burnard, 2000a, 2007; Burnard y Murhpy, 2013; Fautley, 2014; Giglio, 2012; Hickey, 2003; Leung, 2004; Major, 2007; Odena, 2014; Soares, 2012) ha supuesto un acercamiento a su complejidad desde el entorno educativo, otorgando el mismo nivel de importancia tanto al proceso pedagógico como al producto creativo (Burnard, 2000a, 2007; Burnard y Murhpy, 2013; Fautley, 2014; Giglio, 2012; Hickey, 2003; Leung, 2004; Major, 2007; Odena, 2014; Soares, 2012). Así, en el ámbito de la música y la educación musical, la creatividad ha sido descrita como un fenómeno social (Frith, 2012; Miell y Littleton, 2004; Sawyer, 2003), que "emerge en y como prácticas sociales" (Burnard, 2012a, p. 7). A partir de dicha aproximación sociocultural, surgen diferentes formas de creatividad musical (Burnard, 2012b), de carácter fundamentalmente colectivo, que enfatizan aspectos sociales, musicales o culturales.

\subsection{La improvisación Colectiva}

La improvisación colectiva ha representado una práctica constante a lo largo de la historia de la música en un amplio abanico de contextos como, por ejemplo, la música gamelán de Java (Brinner, 2007), la música india, iraní (Nooshin y Widdess, 2006) o japonesa (Prevost, 1995), flamenco (Batista, 2008), jazz (Berliner, 2002) o la música aleatoria (Cage, 1961), entre otras. Asimismo, en el ámbito profesional occidental, una de las propuestas pioneras es la Improvisación Libre desarrollada por Dereck Bailey desde los años 70 del siglo XX, cuyas raíces se podrían situar en el free jazz. No está sujeta a ningún modelo discursivo, sino dinámico, según Mattheus (2012). Surgió como cuestionamiento de un lenguaje, es decir, se pretendía romper los lazos con los enfoques heredados a través de una búsqueda emocional o instintiva que pudiera ser abordada por todo tipo de intérpretes, desde principiantes a virtuosos. El modelo no inspira el contenido, sino la propia interacción, aunque siempre hay influencias discursivas en los intérpretes.

Podemos encontrar dos perspectivas diferenciadas en el término libre aplicado a la improvisación. En primer lugar, idiomático, como ya se ha mencionado; esto significa que no viene determinado por un lenguaje musical concreto marcado por sus propias reglas como en el Jazz o en la música tonal, sino que se construye desde un enfoque experimental. Expresado de una forma simple: no hay reglas. En segundo lugar, jerárquico, ya que no se construyen relaciones de jerarquías ni está sujeta a convenciones, tal y como subraya Alonso (2014). 
Además, cabe mencionar el desarrollo de orquestas de improvisadores dirigidas en experiencias como Globe Unity Orchestra, la London Jazz Composers Orchestra o la Berlin Contemporary Jazz Orchestra; estas, durante los años sesenta y principios de los setenta, comienzan a improvisar desde el free jazz y son dirigidas por algún miembro del grupo (Alonso, 2008). Se trata de una forma de improvisación colectiva en la que un director guía al grupo a través de una serie de signos ideográficos y gestos que constituyen un vocabulario que sirve de comunicación con el grupo de músicos; a partir de este último, se elabora música en tiempo real. Cada signo y gesto transmiten información para la interpretación individual y/o colectiva, por lo que permiten concebir o modificar de forma instantánea la armonía, melodía, ritmo, articulación, fraseo, forma etc.

El germen de esta forma de improvisación se podría localizar en las experiencias de orquestas como la que desde mediados de los años ochenta, el director y compositor norteamericano Butch Morris, considerado una de las figuras clave de esta práctica (Matthews, 2012), define como Conduction. Walter Thompson $(2006,2009)$ fundó su propia orquesta (Walter Thompson Orchestra) en 1984 desarrollando una estrategia de improvisación colectiva dirigida, basada en la utilización de una serie de "gestos corporales"; la denomina Soundpainting y presenta notables implicaciones pedagógicas. Se trata de una forma de improvisación en la que "ciertas decisiones son tomadas por cada músico y otras por un director" (Matthews, 2012, p. 116) que "centraliza el devenir musical y relaciona a los improvisadores" (Alonso, 2008, p. 65).

La investigación sobre improvisación en el aula se ha incrementado notablemente durante los últimos veinte años en España y en el contexto internacional, centrada en aspectos como su relación con la composición (Burnard, 2000b; Peñalver, 2005), los efectos en el pensamiento creativo del alumnado (Koutsoupidou y Hargreaves, 2009), o el análisis de los procesos creativos que en ella se desarrollan (Custodero, 2007). En el caso de investigaciones sobre improvisación colectiva, se han abordado las características propias de la interacción en el jazz (Sawyer, 2008; Seddon, 2004), de aspectos emergentes en relación con la identidad del alumnado (MacDonald, 2014), o del análisis específico de la improvisación libre (Burnard y Dragovic, 2014; Hickey, 2009, 2015).

\subsection{Características de la improvisación colectiva desarrollada en el estudio de investigación}

El germen didáctico de la improvisación colectiva guiada podemos encontrarlo en las propuestas didácticas de Murray Schafer (1965), John Paynter (1970), George Self (1991) o Brian Dennis (1991). Durante la primera década del s. XXI, se han elaborado propuestas con ciertas similitudes en el ámbito educativo, entre otras, las denominadas Soundpainting (Thompson, 2006, 2009) o Creación Sonora en Tiempo Real (Espinosa, 2005). En todas ellas encontramos una esencia didáctica común: una forma de improvisación colectiva guiada.

La improvisación, tal y como se ha desarrollado en el presente estudio, constituye una propuesta didáctica centrada en los siguientes ejes:

- Construcción e interacción conjunta profesor-alumnado con un programa previo.

- Utilización de procedimientos propios de la música contemporánea como la música aleatoria. No obstante, también se sirve de otros estilos musica- 
les, como el Jazz o Pop, partiendo de las preferencias musicales del alumnado.

- Búsqueda de "paisajes sonoros"; actividad muy apropiada para la creación audiovisual desde la perspectiva de las atmósferas sonoras.

- La experimentación sonora como guía didáctica.

- Construcción de experiencias musicales tomando como punto de partida la música y las emociones.

- Utilización de gestos, por parte del docente (guía y animador), que representan sonoridades globales. Se trataba, en definitiva, de buscar entre todos una amplia gama de sonoridades que sirviera de "paleta sonora" para las improvisaciones posteriores.

\section{Método}

Desde nuestra experiencia docente, con una voluntad transformadora (Acaso, 2013) y considerando una triple vertiente centrada en: (a) la mejora de los procesos aprendizaje (Cochran-Smith y Litle, 2009); (b) la metodología, para buscar nuevos enfoques para la música en secundaria (Saunders y Somekh, 2009); y (c) la mejora de nuestra propia práctica docente (Hargreaves y Fullam, 2014), hemos desarrollado un proyecto de Investigación Acción (IA) intradepartamental (McNiff y Whitehead, 2002), convencidos de la importancia de la comprensión de la realidad educativa desde dentro (Burnard, Apelgren y Cabaroglu, 2015), en colaboración con artistas e investigadores educativos.

La investigación se desarrolló en 5 ciclos a lo largo de 6 cursos académicos, desde 2007/2008 hasta 2012/2013 y han tomado parte 11 grupos de $3^{\circ}$ de ESO, integrados por un total de 267 alumnos y alumnas en tres institutos de Educación Secundaria de las localidades madrileñas de Rivas Vaciamadrid y Arganda del Rey, en contextos heterogéneos desde una perspectiva socioeconómica e intercultural, con alumnado de más de 10 nacionalidades, o diversas creencias religiosas.

A lo largo de los ciclos se implementaron una serie de proyectos didácticos, centrados en la creación musical a través de un soporte visual, y caracterizados por su constante evolución. Es decir, el alumnado musicalizó carteles o cuadros y compuso bandas sonoras a través de la composición cooperativa y/o la improvisación colectiva como estrategias didácticas.

Los procesos de planificación y preparación de cada uno de los ciclos, desde el punto de vista temporal, fueron similares: se consideró la organización de los cursos escolares de Enseñanza Secundaria en tres trimestres que transcurrieron desde mediados de septiembre hasta finales de junio, con dos períodos vacacionales en Navidad y Semana Santa. Los citados trimestres se correspondían, con ligeras diferencias entre los distintos centros, con tres evaluaciones. Los procesos de planificación y preparación de los proyectos se desarrollaron durante el primer trimestre y la primera parte del segundo. La implementación de los mismos tuvo lugar desde la segunda parte del segundo trimestre hasta el final de curso.

El estudio propuso la comprensión de los procesos de aprendizaje del alumnado en la creación musical, como herramienta para la transformación educativa en el aula. Todo ello a través de una comprensión transversal de la participación en el aula, tanto desde la perspectiva investigadora (Heron y Reason, 1997), como educa- 
tiva (Martínez, 1998), y a través de un eje conjunto con la creación musical. De este modo, los objetivos planteados en esta investigación tomaban como punto de partida la mejora de la comprensión de los procesos de aprendizaje del alumnado a través de la puesta en práctica de un proyecto didáctico. Así, se concretaban en examinar las características del mismo en relación con la motivación y las creatividades musicales, tanto desde la perspectiva del aprendizaje creativo como del aprendizaje para la creatividad, además del análisis de las estrategias didácticas desarrolladas en el aula y su idoneidad en el ámbito de la Educación Secundaria.

\subsection{El Proyecto Pintando Sonidos}

El presente artículo aborda la comprensión en profundidad de la improvisación colectiva como estrategia didáctica en Educación Secundaria a través del análisis pormenorizado del cuarto ciclo del estudio, puesto que se centra en la musicalización de una selección de cuadros de una pintora de reconocido prestigio a través de la citada estrategia didáctica. Así, las preguntas de investigación plantean la comprensión de los procesos de aprendizaje y los aspectos emergentes durante el proyecto didáctico, así como en el análisis de la utilidad de la improvisación colectiva en la Educación Secundaria.

\subsubsection{Contexto del estudio y participantes}

Este estudio se llevó a cabo en un instituto público de Rivas Vaciamadrid, localidad situada al sureste de la Comunidad de Madrid. El alumnado fue heterogéneo, con un ambiente positivo de relación entre iguales, y sin problemáticas de conducta o disciplina de especial gravedad, además de un interesante grado de implicación y satisfacción en el centro. La relación profesor alumnado es, en general, cordial y agradable. Muchos alumnos se dirigen al profesor por su nombre de pila, o junto con el coloquial "profe", muy habitual.

La investigación se desarrolló con un grupo de tercero de ESO cuya elección se debió a razones pedagógicas, para facilitar el desarrollo del proyecto, así como a razones organizativas para que el profesorado responsable de la investigación pudiera estar presente en las distintas sesiones. Se trataba de un grupo heterogéneo, tanto desde el punto de vista de resultados académicos como actitudinal y de comportamiento en el instituto, que, les gustaba mucho hacer música en clase, como señalaron en las entrevistas, y poseían, casi sin excepciones, un buen auto-concepto con respecto a sus habilidades musicales, observando además, que una gran mayoría del alumnado se consideraba en el promedio en referencia a su clase. Por tanto, la predisposición hacia propuestas didácticas centradas en lo procedimental en el área de Música, parecían evidentes y resultaron ser un factor importante que influyó de forma positiva en el desarrollo del estudio. Igualmente, es importante señalar que el grupo no había desarrollado con anterioridad, tanto en Primaria como en Secundaria, propuestas didácticas centradas en la creatividad a través de la improvisación o composición musical.

\subsubsection{Recogida de datos}

En el desarrollo de esta investigación se emplearon diferentes técnicas de recogida de datos: (a) observación participante; (b) observación no participante; (c) grabaciones audiovisuales; (d) entrevistas y (e) encuestas, a través de diversos instrumentos como: 
(a) diario de clase; (b) planilla de recogida de las declaraciones de entrevistas; (c) informes de observación; (d) registro de análisis de las grabaciones audiovisuales.

Desde la concepción del profesor investigador se realizó la observación participante, plasmando tras cada una de las sesiones, la evolución, actitud, progreso y pensamientos de cada momento en un diario de clase. La observación no participante fue llevada a cabo por la jefa de Departamento observando alguna de las sesiones, así como viendo en vídeo todas las sesiones, que posteriormente, analizamos de forma conjunta. Además, se realizaron entrevistas semiestructuradas a tres grupos de alumnos para obtener su perspectiva sobre los procesos de aprendizaje, que se completó con un cuestionario de tipología abierta y cerrada. También, hemos recabado la perspectiva de la pintora, mostrándole ejemplos de los resultados obtenidos. Finalmente, todo ello se ha realizado en colaboración con dos profesores del Departamento de Expresión Musical y Corporal de la Facultad de Educación de la UCM y University of Eastern Finland, quienes han validado el diseño y análisis del mismo.

\subsubsection{Procedimiento del proyecto didáctico}

En la planificación del proyecto se partió de la experiencia anterior en los ciclos previos de investigación desarrollados por el profesor del grupo (Lage y Cremades, 2017), y centrados en la creatividad a través de la creación cooperativa y de la improvisación colectiva. Así, el proyecto surgió debido a dos motivaciones básicas:

- $\quad$ El desarrollo de nuevos proyectos didácticos adaptados al estilo de aprendizaje musical del alumnado que potenciaran el pensamiento creativo, colectivos, que promovieran la creación de experiencias musicales significativas en el aula y motivadoras para el alumnado. Todo ello ante la comprobación de su desmotivación ante los procesos de enseñanza-aprendizaje que podríamos calificar como tradicionales, y centrados en el conocimiento declarativo.

- La necesidad de la investigación en el aula como herramienta para la mejora de los procesos de enseñanza-aprendizaje y de nuestra práctica docente.

En octubre de 2010 el Departamento de Música planteó el desarrollo de un proyecto de IA junto con una pintora, que consistió en la musicalización de una selección de sus cuadros a través de la improvisación colectiva como estrategia didáctica.

En primer lugar, contactamos con una pintora profesional de reconocido prestigio. La artista se mostró muy receptiva y escogió una serie de cuadros que le parecieron idóneos para el trabajo que le estábamos planteando. Sin embargo, y aunque la visita a su estudio fue fundamental para contemplar los cuadros in situ, estos, por diversos motivos, no pudieron ser trasladados al instituto. Durante el proyecto, el alumnado pudo visualizar los cuadros proyectados en la pizarra digital del aula.

Durante las entrevistas con la pintora se produjo un acercamiento a las características estilísticas de su obra, que elabora desde la intuición artística a través de la manipulación, transformación de diversos materiales (libros desechados u otro tipo de restos industriales) y la repetición de secuencias, dotando a su obra de una nueva carga conceptual. 
"Cuando hablo sobre mi trabajo, lo comparo con escuchar música o danzar, para no racionalizarlo o buscar una narrativa, simplemente sentirlo" (Pintora, Entrevista)

Se constata una importante perspectiva emocional en su trabajo, que resultó útil para canalizar nuestras propias musicalizaciones, tanto desde el punto de vista del proceso de aprendizaje del alumnado, como su relación con la creatividad y el ambiente del aula (Odena, 2012). Desde una perspectiva didáctica, los objetivos del proyecto fueron los siguientes:

- Potenciar el pensamiento creativo del alumnado a través de la creación musical vinculada a su vez con la pintura, en ambos casos con lenguajes contemporáneos, para favorecer la comprensión de un lenguaje artístico del que habitualmente se sienten alejados.

- Familiarizar y acercar a los estudiantes a la creación contemporánea, y hacerlo desde una perspectiva transversal, global e integradora, con la pretensión de involucrarles en el arte contemporáneo desde la realidad de obras de reciente creación, y con cuya autora pudieran compartir experiencias que facilitaran la musicalización posterior.

- Incrementar la motivación y la participación del alumnado, favoreciendo la consecución de experiencias musicales significativas.

- Desarrollar las habilidades expresivas e interpretativas por parte del alumnado.

- Conferir un papel activo en su propio aprendizaje.

Durante los dos primeros trimestres del curso, realizamos una reflexión previa sobre los estilos de aprendizaje del alumnado a través de la observación participante. Además, se administró un cuestionario inicial relacionado con el aprendizaje musical. Este fue validado por profesores del departamento de Métodos de Investigación y Diagnóstico en Educación, así como de Expresión Musical y Corporal de la UCM. Todo ello, con el objetivo de corroborar sus preferencias; la perspectiva sobre su propio aprendizaje, capacidad de abstracción o concreción, de cuándo y dónde preferían aprender, y de cómo aprendían en grupo. A partir de dicho análisis, se desarrolló y concretó el proyecto didáctico para acercarlo a los estilos de aprendizaje del alumnado, e intentando que, a través de la actividad, su acercamiento al mismo fuera diverso en función de cada alumno/a.

En primer lugar, y como aspectos éticos de la investigación, se expuso la posibilidad de realizar una investigación al alumnado implicado y a la pintora. Tras su respuesta satisfactoria se solicitaron los permisos pertinentes de la dirección del centro y de la jefatura de estudios. Asimismo, se informó y solicitó el permiso a las familias para la grabación en vídeo y la toma de imágenes.

La recogida de datos se llevó a cabo durante el tercer trimestre del curso 2010/2011 a lo largo de 14 sesiones: La primera se dedicó a mostrar y explicar la obra de la pintora, así como a seleccionar los cuadros que íbamos a musicalizar. Durante las dos siguientes sesiones, se produjo la toma de contacto con la estrategia didáctica a través de la asociación de los distintos gestos con sonoridades específicas. Se trataba, en definitiva, de buscar entre todos una amplia gama de sonoridades que sirviera de "paleta sonora" para las improvisaciones posteriores. Siguiendo a Thompson (2006), se utilizaron distintas gestualidades en función de: (1) Qué tocar (2) Cuándo tocar (3) Cómo tocar y (4) Quién 
va a tocar. Así, las dos primeras sesiones fueron sistemáticas desde el punto de vista del trabajo específico sobre la gestualidad y sonoridad específica del grupo. Pretendíamos buscar una sonoridad propia en la que el alumnado se sintiera integrado, similar al trabajo en una orquesta. Y en ese sentido, el papel participante del docente era obvio.

En estas dos primeras sesiones de toma de contacto y empaste, se produjeron algunos momentos tediosos por la necesidad de aprendizaje por parte del alumnado de la gestualidad. Se trataba, en definitiva, de dotar al alumnado de herramientas para las improvisaciones posteriores que posteriormente se mezcló con las del propio del alumnado.

La sonoridad de los gestos tiene similitudes con la música aleatoria, serial o minimalista de las vanguardias de la segunda mitad del s. XX. Sin embargo, cuando comenzamos con las improvisaciones de los cuadros, emergieron sonoridades propias de la mochila musical del alumnado, donde encontramos elementos de la música Pop o Jazz. Así, se produjo una fusión estilística muy variada, que ayudó a familiarizar al alumnado con otras músicas.

Posteriormente, comenzamos el trabajo específico con los cuadros que el grupo había seleccionado. Al igual que con la pintora, no establecimos una teorización excesiva sobre la música y los cuadros, excepto algún comentario explicativo. Buscábamos sonoridades desde la intuición. Y fue así cómo se produjo progresivamente la transformación del aula. Dos alumnas propusieron elaborar textos sobre los cuadros para después recitar o leer, y aprovechamos además el uso de palabras que sugerían los cuadros como elemento para las improvisaciones. Junto a la voz, los instrumentos que utilizamos fueron: teclados, batería, guitarras, trompeta, congas, bongós y otros de pequeña percusión junto a metalófonos, xilófonos y carrillones.

La organización espacial del aula e instrumentos representaba otro elemento importante. Para ello, se planteó un espacio abierto en forma de U en el que el alumnado eligió los instrumentos, incluyendo la voz, disponibles en el aula, o bien aportando instrumentos propios que estudiasen de forma extraescolar. Todo ello con el objetivo de crear un clima que favoreciera la labor en la que nos íbamos a adentrar. Pero tras la planificación nos asaltaba una duda, ¿cómo iba a ser el proceso de aprendizaje a través de una estrategia novedosa para el alumnado?

\section{Análisis y exposición de los datos}

El marco analítico del estudio se ha desarrollado a través de un "proceso iterativo mediante la lectura y relectura de los datos" (Rice y Ezzy, 1999, p. 258) así como mediante la organización, revisión y reflexión de los mismos con el objetivo de su mejor descripción (Kvale, 1996). En este sentido, se ha establecido una clasificación en cuatro dimensiones generales de análisis interconectadas entre sí y vinculadas con las preguntas de investigación, en las que se han analizado aspectos, tanto particulares como generales, para favorecer, entre otras, la descripción densa (Geertz, 1988) del fenómeno estudiado.

\subsection{El proceso creativo de aprendizaje en el proyecto didáctico: el fluir colectivo}

Consideramos especialmente significativo cómo el proyecto se ha desarrollado a través de un proceso constante, aunque con altibajos, donde la implicación y actitudes 
del alumnado, junto a los resultados musicales, iban mejorando de forma ascendente y gradual.

"Ha habido momentos, al principio, algo caóticos, por la inseguridad que generaba no saber qué hacer. Ante ello, he tenido que llamar la atención una vez y proponer que estructuraran la pieza en torno a un estribillo. Poco a poco, han ido solventando la situación y su actitud e implicación mejoraban. He sugerido, además, que todos intervinieran en todas las secciones de la pieza. El ambiente del aula ha ido mejorando a medida que se sentían más seguros y, por tanto, su atención se mantenía fija en la actividad" (Diario de clase, Profesor)

"Veíamos como las cosas salían poco a poco" (Entrevista, 15 de junio de 2011, Alumno 1)

Csikszentmihalyi (1998, p. 139) define el fluir como una "experiencia óptima" durante el proceso creativo. Explica cómo la experiencia del fluir se puede explicar a través de 9 características independientemente de la actividad que la produzca: (a) metas claras (b) respuesta inmediata a las propias acciones (c) equilibrio entre dificultades y destrezas (d) concentración (e) centralidad de la actividad durante el proceso (f) ausencia de miedo al fracaso (g) desaparece la autoconciencia (h) distorsión del tiempo (i) la experiencia como centro en sí misma o autotélica. Sawyer (2003) desarrolla el concepto análogo a nivel colectivo. Resulta muy complejo de explicar, pero se trata de experiencias aglutinadoras que se reflejan a través de emociones positivas o placenteras y que el grupo experimenta colectivamente.

En el contexto de la investigación, la triangulación de los datos revela cómo a partir de la sesión 7 se ha denotado un punto de inflexión en el proyecto con una mejora significativa del clima del aula, y en el que se corroboran las características que explican la experiencia del fluir, en este caso a nivel colectivo.

"Tengo la sensación de que hay un momento de "clic", a partir del cual todo parece que comienza a funcionar mucho mejor" (Diario de clase, Profesor)

"Hoy ha habido momentos muy interesantes en las improvisaciones, he sentido emoción e inspiración" (Diario de clase, profesor).

El alumnado se involucró significativamente a partir de su identificaron con el proyecto, es decir, hicieron propia la música que estaban creando y su vínculo con los cuadros como metas del mismo. La progresiva satisfacción con sus propias improvisaciones resultó un elemento esencial en el progreso del proyecto, tanto desde el punto de vista de los productos musicales, con un incremento del nivel de auto exigencia en las ejecuciones musicales, como desde su grado de satisfacción.

Tras una improvisación de 5 minutos, un alumno dijo: ¡Cómo mola!, ¡sí!, continuaba otro grupo de alumnado. El resto de la clase asentía con aplausos (Sesión 7, transcripción de la sesión) 
Se generaron una serie de emociones positivas que se pusieron de manifiesto a través de las sinergias producidas entre el grupo y con el profesor. En ese momento el alumnado se reafirmó en sus habilidades musicales y el contexto de la clase dejó de ser el del aprendizaje habitual en un aula de Secundaria. En ese momento se convirtieron en músicos, con un grado de concentración muy elevado, cuyo aprendizaje se estaba produciendo de forma intuitiva, transformándose así su relación con la música.

"El alumnado destaca el papel del profesor como guía en estas actividades. Han considerado especialmente significativa su constancia, ayudándoles a "sacar lo mejor" de ellos mismos. En definitiva, la experimentación sonora ha permitido la construcción de nuevos contenidos musicales, artísticos y actitudinales sobre lo ya aprendido previamente. Además, hay que subrayar que a través de la improvisación colectiva, se ha incrementado notablemente su respeto por la música". (Entrevista, 21 de junio de 2011, observadora no participante)

En dichas experiencias, el conjunto es más importante que la suma de sus individualidades, algo que se podría identificar con el concepto de comunión, desde su acepción de participación en algo común. A través de los procesos de aprendizaje en la improvisación colectiva, se ha conformado la identidad del grupo. El alumnado asumió con satisfacción su rol en el grupo, más allá de su mayor o menor participación según sus habilidades musicales o el instrumento o instrumentos que hubieran tocado, ya que lo importante era formar parte de un conjunto que estaba improvisando de forma colectiva y donde la aportación de todo el grupo era fundamental (Sawyer, 2008). Observar cómo el alumnado proseguía con las improvisaciones hasta su conclusión, que no siempre coincidía con el timbre que marca el final y bastantes veces se alargaba, pone de manifiesto la implicación y satisfacción con el proyecto didáctico.

"El alumnado ha estado envuelto en sonido, en su sonido y, como consecuencia, esta experiencia les ha conducido más allá de la propia música, convirtiéndoles en creadores de algo común. Se les veía entusiasmados" (Entrevista, 21 de junio de 2011, observadora no participante)

La triangulación de los datos refleja cómo el alumnado participó en función de sus habilidades musicales y construyó su aprendizaje desde cuatro ejes: (1) la experimentación propia con el instrumento, (2) las propuestas y sugerencias del profesor, (3) el intercambio de ideas con el grupo en su conjunto desde la sonoridad colectiva y (4) de las aportaciones particulares de otros compañeros.

\subsection{La voz del alumnado a través de la improvisación colectiva}

El análisis de los productos musicales en las improvisaciones colectivas se realizó de forma conjunta entre el profesor investigador y la observadora no participante a través del análisis videográfico de todas las sesiones. Dicho análisis se focalizó en definir y categorizar las características musicales las improvisa- 
ciones: duración, estructura, material motívico y estilo musical. Todo ello fue triangulado con el diario de clase del profesor investigador y las entrevistas y cuestionarios al alumnado.

- Duración: La duración de las improvisaciones ha ido evolucionando durante el proyecto. De forma paulatina se fue incrementando desde 1 a 5 minutos durante las primeras 7 sesiones, hasta los 35 minutos de las sesiones 11, 12 y 13.

- Estructura: Esta venía determinada por el establecimiento previo de desarrollo dramático como relajación-tensión-relajación o tensión-relajacióntensión, enmarcadas en las estructuras clásicas de $\mathrm{ABA}, \mathrm{AB}$, o similares.

- Material motívico: Algunos de los aspectos reseñables serían:

1. Paletas sonoras, en ellas se muestra la influencia de la cultura musical del alumnado.

2. Improvisación libre, emergente en el momento de tocar; en ella, de igual forma, se observa la influencia de la cultura musical del alumnado.

3. Los efectos sonoros como atmósferas o paisajes sonoros.

4. La experimentación sonora.

El análisis de los productos nos remite, a su vez, a la relación entre música e imagen. En este sentido, salvo la utilización de textos y palabras que el cuadro le evocaba al alumnado, sería muy complejo encontrar vínculos directos. Sin embargo, resulta evidente que marcaba el desarrollo de las improvisaciones, pero desde una perspectiva implícita y basada en las asociaciones entre la música y la imagen, que podrían considerarse de carácter cultural y que en el presente estudio hemos podido corroborar.

Hemos denotado aspectos de enculturación e identitarios desde el punto de vista de los estilos musicales utilizados, tanto de forma explícita como implícita (Gordon, 1996; MacDonald, Hargreaves y Miell, 2017). Ya que se ha establecido, como se ha comentado, una negociación implícita, donde emergen músicas muy variadas, desde las "mochilas musicales del grupo", que muestran la emergencia citada. Nos referimos a: los elementos vanguardistas procedentes de la segunda mitad del s. XX a través de atmósferas sonoras o elementos de aleatoriedad; ritmos propios del rock, jazz o del minimalismo propio de las bandas sonoras. Todo ello se realiza de forma ecléctica, pero, como ya se ha mencionado, identificado por el alumnado como propio, y que se podría definir como una forma de caleidoscopio cultural.

Cuando el alumnado ha tomado contacto con el contenido inherente, el lenguaje musical debido a la experiencia en la improvisación colectiva, más allá de los contextos sociales estandarizados y externos al aula, se ha generado una nueva delineación (Green, 1988). Gracias, entre otras, a su propia creatividad, su música se ha hecho autónoma a través de un proceso de experimentación musical auténtico. Se han establecido, así, nuevos significados delineados desde la toma de contacto con los contenidos inherentes adaptados a las habilidades o necesidades del alumnado.

Cuando el alumnado autoevalúa los progresos en su pensamiento creativo, en general consideran que han sido notables, reflejándose en la capacidad para crear obras propias que han experimentado, tanto individual como colectivamente. 


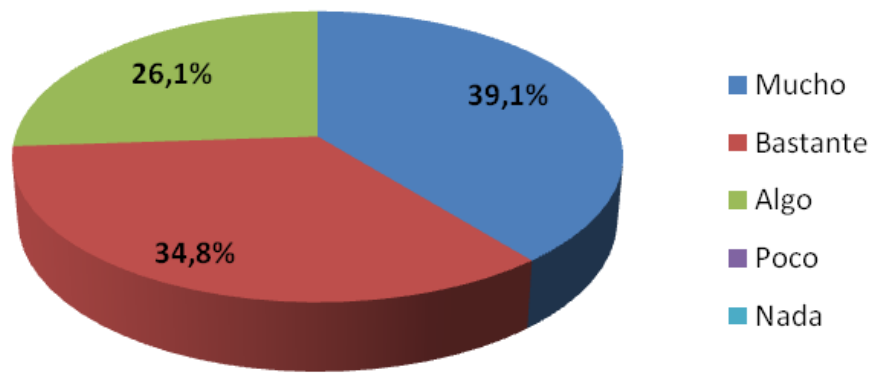

Figura 1. Percepción del grado de mejora del alumnado en creatividad musical

El 39.1\% del alumnado ha respondido "mucho", 34.8 "bastante", mientras que el $26.1 \%$ cree que ha aprendido algo. Los motivos expuestos se basan en el desarrollo producido a partir de la improvisación colectiva, que ha favorecido la capacidad para crear obras propias, y la valoración de la actividad en la faceta de la expresión libre y espontánea. Además, han vinculado la experimentación de nuevos aprendizajes con una gran motivación. Han manifestado cómo ésta ha mejorado desde la práctica instrumental, además de descubrir sus propias posibilidades, llegando a resultados que no podrían haber sido experimentados de antemano. Como consecuencia, expresan su satisfacción por el descubrimiento de sus posibilidades que, procedentes de los avances en práctica instrumental, les condujo a resultados significativos. Si bien algún estudiante reconoce que aún podría haber aprendido más, la valoración positiva de la improvisación y la expresión libre y espontánea es generalizada. Fragmento del diálogo entre cuatro alumnos en una entrevista semiestructurada:

"Siempre se oía algo diferente y eso era lo que nos gustaba, por lo menos a mí, crear algo nuevo, que no sabías lo que iba a pasar y con la incertidumbre de qué ibas a hacer" (Entrevista, 10 de junio de 2011, alumna 2) "Tenían motivación porque veíamos que salía y que sonaba bien" (Entrevista, 14 de junio de 2011, alumno 3) "Todos hemos estado motivados con lo que nos ha tocado, por ejemplo Diego con el piano, Ana con la trompeta, Jesús con el teclado y yo con las láminas" (Entrevista, 15 de junio de 2011, alumno 4) "Crear algo propio siempre gusta más que copiarlo" (Entrevista, 10 de junio de 2011, alumna 2)

En el análisis conjunto con la observadora no participante tras la visualización de los vídeos en relación con los aprendizajes del alumnado, señalábamos el avance de la expresividad, atención, musicalidad, la importancia del silencio y de saber escuchar gracias a la actividad, mediante las interpretaciones y el clima agradable y de confianza mutua entre el alumnado, como estímulo para la participación, experi- 
mentación y búsqueda de nuevas sonoridades. El desarrollo del proyecto ha puesto de manifiesto una mejora en la fluidez, en la variedad de nuevas ideas musicales y en la improvisación, construidas desde su experiencia previa, y a través de la ayuda y colaboración del docente, así como del propio grupo, de forma colectiva, que ha estimulado y guiado dicho proceso.

\subsection{Aspectos emergentes en el proyecto didáctico}

Una de las preguntas de investigación que ha guiado el proceso de indagación ha consistido en analizar la motivación del alumnado en los diversos proyectos didácticos. A lo largo del estudio se ha puesto de manifiesto la relevancia en su aprendizaje de los procesos emergentes en relación con la motivación, el ambiente del aula, las emociones positivas, así como el bienestar y la democracia o justicia social en el aula, en el marco de un proceso constante de participación.

\subsubsection{Emociones en el aula}

Uno de los temas emergentes a lo largo de la investigación ha sido cómo han surgido emociones positivas en el aula, tanto en el plano individual como social (Chóliz y Gómez, 2002), en relación con la creatividad. A través de la triangulación de los datos, hemos sido conscientes de cómo han surgido a través de los procesos de aprendizaje. Este tema ha sido abordado en el ámbito de la educación musical por Odena (2014) o Veloso y Carvalho (2012), entre otros. Así, nos hemos preguntado, en primer lugar, sobre los motivos por los cuales se han generado y hemos inferido, tras el proceso de reflexión sobre los datos, los siguientes aspectos:

1. La identificación con su propia música

2. La consideración de experiencia musical significativa en el aula, como ha definido una gran parte del alumnado a los proyectos didácticos.

3. El compromiso con el proyecto didáctico como una meta compartida por el grupo, "su proyecto", vinculado al desarrollo de la concepción de uno mismo y de grupo, así como de la participación como expresión conjunta y simbólica de un grupo humano.

4. El ambiente positivo generado en el aula.

Desde la consideración de la música como un vehículo natural de transmisión emocional (DeNora, 2000), hemos apreciado cómo las emociones se han desencadenado a lo largo de los proyectos didácticos de forma transversal, puesto que ellas también se refieren a aspectos identitarios, sociales, etc. Es decir, se han desarrollado desde puntos de vista interconectados: las colectivas del grupo, las individuales del alumnado, también la del profesor. Así, se ha hecho patente cómo han emergido emociones colectivamente durante los procesos creativos como resultado de: "sentirse parte de", identidad, construcción simbólica de una identidad compartida en torno a su propia música.

Las emociones positivas se han detectado en el aula a través de las siguientes situaciones de aula: 
1. En la interpretación y, de forma explícita, en la expresividad de la música interpretada por el alumnado, es decir, las emociones transmitidas que ha asumido el alumno como propias.

2. Otro ámbito podemos situarlo en las emociones positivas desencadenadas en la clase, derivada del ambiente del aula, relajado, seguro y distendido, de bienestar.

3. El fluir en las interpretaciones colectivas, que, junto con el ambiente emocionalmente seguro para el alumnado y la música en el aula, ha sido un catalizador de emociones positivas: "Nos hemos emocionado con lo que hemos hecho y el recuerdo de dicha música aún nos emociona". Así, la creación de un ambiente como el que se ha dado ha constituido un factor previo para el fluir colectivo.

Algunas situaciones que denotan la importante carga emocional de los proyectos didácticos han sido la manera en que el alumnado ha manifestado los gratos recuerdos que guardaba sobre estos. Se trata de momentos que quedan en la memoria colectiva como especialmente placenteros y que permiten establecer vínculos emocionales que se afirman en comentarios del alumnado como:

"Echamos de menos las clases, nos sentíamos muy bien" (Entrevista, 15 de noviembre de 2011, alumna 6)

Comprobar además la implicación y el desarrollo emocional y la cohesión grupal.

Fragmento de la sesión 13: una alumna canta un tema de despedida (que se había preparado como sorpresa para el grupo). Se generó una notable emoción en el resto de compañeros: aplausos, y abrazos en el grupo.

En definitiva, el grupo se encontraba cómodo y relajado, algo que propició climas apropiados para el aprendizaje musical y para un momento tan interesante desde la perspectiva emocional como el que se produjo. A través de los elementos nombrados al principio, la música dejó de ser considerada como una asignatura en el mismo plano que las demás, se situó en otra categoría, con condicionantes positivos, aunque también negativos.

\subsubsection{Motivación y ambiente del aula}

El proyecto didáctico nos planteó una serie de interrogantes e incertidumbres iniciales ya que la Improvisación Colectiva como estrategia didáctica requiere la implicación, actitud positiva y colaboración del alumnado (Seddon, 2012). Podemos afirmar que el alumnado permaneció durante todo el proyecto con un elevado nivel de motivación, reflejado en sus actitudes individuales y colectivas, en sus declaraciones, y el entusiasmo con el que emprendían todas las actividades propuestas, así como en la participación atenta y creativa que se reflejaba en las sesiones en las que se desarrolló este trabajo. Por otra parte, su empeño cuando se proponía repetir algo para mejorarlo. Estaban profundamente involucrados, tanto en la ejecución instrumental, como en la toma de decisiones consensuadas, bien fuera la elección de los cuadros 
o ritmos y melodías que juzgaban adecuados. Su participación también abarcaba el planteamiento de dudas o recordatorio activo de gestos.

A modo de ilustración, recogemos el comentario de un alumno que expresa su entusiasmo ante la clase de música:

"Por la satisfacción del trabajo con los compañeros y la creatividad que se respiraba en el ambiente" (Entrevista, 12 de junio de 2011, alumno 7)

O cómo la observadora expresaba:

"Se les ve emocionados y entusiasmados" (Entrevista, 21 de junio de 2011, observadora no participante)

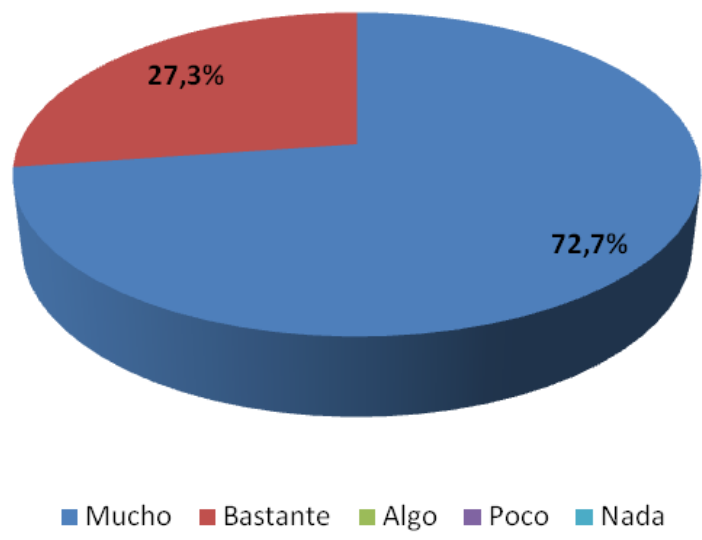

Figura 2. Nivel de motivación

De la encuesta realizada, se refleja cómo el $72.7 \%$ de los y las alumnos/as estaban "muy" motivados y el 27.3\% "bastante". Es decir, el 100\% de la clase manifiesta un nivel de motivación muy elevado.

El alumnado ha sido consciente que su actitud era fundamental para el proyecto, $y$, en general, afirman que fue muy buena, pese a algún que otro comentario en el sentido de que, a veces, les costaba entrar en la tarea musical, porque hablaban demasiado. Sin embargo, observándolos, resultaba conmovedor que, una vez en situación, mantuvieran un silencio previo a los comienzos y mostrando gran concentración. Los motivos se podrían encontrar, entre otros, en la satisfacción con las actividades propuestas. Han valorado positivamente el buen ambiente, la importancia que ha tenido para ellos expresar, de un modo libre y en la práctica, muchos de los parámetros musicales que conocían o estaban aprendiendo sobre la marcha. 


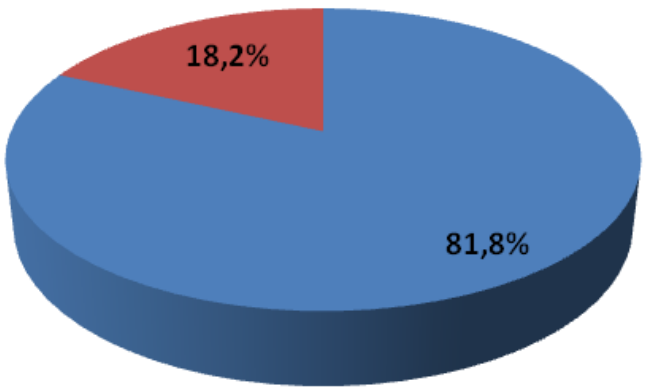

Muybueno $\quad$ Bueno $\quad$ Regular $\square$ Malo $\square$ Muy Malo

Figura 3. Valoración sobre el ambiente en la clase

El alumnado reflejó su percepción sobre el ambiente de clase en la encuesta de forma nítida: toda la clase ha considerado el ambiente durante el proyecto "bueno" $(81.8 \%)$ o "muy bueno" (18.2\%).

\section{Implicaciones para la práctica docente en Secundaria}

Según Matheus (2012), en la improvisación no existen diferencias entre el proceso y el producto: el proceso es el producto, por lo que podríamos considerar la improvisación como un amplificador de los procesos compositivos, es decir, como una forma de hacerlos audibles. En el proyecto, se podrían diferenciar, tomando como referencia el modelo de Peñalver (2005), las siguientes fases en el proceso creativo, que se sucedían de forma cíclica:

1. Toma de contacto con la improvisación colectiva como estrategia didáctica a través del aprendizaje de la variedad de sonoridades que representaban los diferentes gestos (indicaciones del profesor-investigador):

Fragmento sesión 4: Fijaos, hoy empezaremos con el puntillismo. Lo marcaré así. Se trata de tocar muchísimas notas en vuestros instrumentos, una detrás de otra, como si quisierais tocar con las manos todas las estrellas del cielo lo más rápido posible. Marco y empezamos. A ver? " (Suena). Tenemos que intentar que el sonido sea más ligero. Más así. Ejemplificación con el piano y mientras suena, se gradúan los distintos planos dinámicos. Así, muy bien, chicos

2. Estímulo en forma de imagen. Tras el proceso inicial de selección de los cuadros y de explicación de sus características, desde el punto de vista de la autora y de su proceso creativo, buscamos, de forma colectiva, posibles significados conjuntos, a partir de una lluvia de ideas en forma de palabras que nos evocan ellas. 
3. Interpretación y reflexión. La música surgía directamente de los gestos y del imaginario colectivo, tras el que se producía un proceso de reflexión en el grupo, y así sucesivamente.

Además, se podrían establecer una serie de implicaciones para la práctica de la improvisación colectiva en el aula de Secundaria:

- Aproximación a la improvisación desde el ensayo-error como estrategia creativa.

- La importancia del silencio y la concentración como punto de partida del proceso creativo.

- Espíritu de grupo, identidad colectiva: se trata de una creación musical que se produce de forma colectiva.

- La atmosfera o el ambiente como elemento determinante en los procesos creativos y productos.

- Emoción y sentimiento a través de sonido por medio del fluir colectivo en la búsqueda de experiencias musicales significativas.

- Los instrumentos musicales y/o la voz se convierten en un medio para la expresión de la música.

- Las peculiaridades propias del contexto como punto de partida: el contenido musical de las improvisaciones como elemento de enculturación

- $\quad$ Espontaneidad e inmediatez como elementos esenciales para establecer una pulsación propia.

\section{Conclusiones}

El proyecto didáctico se desarrolló como una herramienta para desencadenar un proceso de transformación del aula, tanto desde la perspectiva del aprendizaje como de la enseñanza, y a través del eje transversal formado por la creación musical y la participación activa del alumnado, entendida desde una visión didáctica e investigadora.

Para todo el alumnado, el proyecto didáctico en el que han participado ha supuesto su toma de contacto con la creación musical. Por tanto, partiendo de dicha premisa, la imagen ha representado una guía compositiva, un apoyo en un proceso complejo que, desde la perspectiva didáctica, se ha centrado en la experimentación como estrategia compositiva. Además, como una fuente de motivación, dado el contexto audiovisual en el que nos encontramos (INJUVE, 2012), y como una aproximación integral al hecho artístico. Desde esta perspectiva, nos ha parecido más adecuada la utilización del término "Proyecto Transversal" como característica definitoria. El motivo estriba en que, pese a que han estado aglutinados en la creación musical, se ha desarrollado en conjunción con la pintura. Dicha denominación podría resultar de utilidad en el contexto de la Educación Secundaria, debido a las características propias de su organización curricular.

Por otra parte, hemos podido constatar el desarrollo del pensamiento creativo, la expresividad, atención, "musicalidad", la importancia del silencio y de saber escuchar, a través de las interpretaciones y mediante un clima agradable y de confianza mutua entre profesor y alumnado como estímulo para la participación, experimentación y búsqueda de nuevas sonoridades. En este sentido, el alumnado ha destacado 
el papel del profesor como guía en estas actividades, considerado especialmente significativo su constancia, ayudándoles a "sacar lo mejor" de ellos mismos. En definitiva, la experimentación sonora ha permitido la construcción de nuevos contenidos musicales, artísticos y actitudinales sobre lo ya aprendido. Además, hay que destacar que, a través de la Improvisación Colectiva, se ha incrementado notablemente su respeto por la música.

Una característica fundamental para entender el trabajo en este caso colectivo ha sido la importancia de la naturaleza y la cualidad de la interacción en sí misma, entendiendo el aula como una realidad social compartida. Asimismo, en la creación musical, se han denotado canales de comunicación verbal y otros exclusivamente musicales. Es decir, la improvisación colectiva se ha convertido en un vehículo para la socialización del grupo más allá del aprendizaje musical en sí mismo.

Igualmente, el alumnado ha comprendido la importancia del proceso como parte de la evaluación. Han hecho hincapié en que se valoraba su esfuerzo y participación, siendo conscientes de haber mejorado musicalmente, y ponen de manifiesto, que el proyecto ha culminado con un elevado grado de alcance de las expectativas que en él tenían depositadas, circunstancia que ha fomentado la construcción de experiencias musicales significativas.

Para continuar, basándonos en las preguntas que nos planteábamos como eje vertebrador de la investigación, podemos afirmar que los aprendizajes musicales han resultado reforzados por una motivadora práctica, aglutinadora de contenidos de distinta índole que van desde los parámetros musicales y los elementos inherentes a este lenguaje, a los contenidos curriculares de carácter histórico-estilístico, dentro de un contexto estético, en el que el aprendizaje se ha producido a través de la experimentación sonora como procedimiento compositivo fundamental a través de: (a) la experimentación propia con el instrumento; (b) las propuestas y sugerencias del profesor; (c) el intercambio de ideas con el grupo desde la sonoridad colectiva; y (d) de las aportaciones particulares de otros compañeros. Todo ello con el vínculo entre el desarrollo del pensamiento creativo y las emociones, englobando la expresividad interpretativa, implicación en el proyecto, clima del aula y motivación.

La Improvisación Colectiva se ha mostrado como una útil y versátil herramienta didáctica en la que los alumnos se han visto envueltos en un proyecto dinámico, que potencia sus capacidades y permite una expresión libre. El clima de confianza, la consciencia de posibilidades antes impensables, la mayor audacia expresiva en un contexto en el que las producciones propias son admitidas, estimuladas y valoradas dentro del grupo, donde se intercambian papeles y perspectivas, genera dinámicas que han llevado a todos los participantes hacia nuevas experiencias, partiendo del aprendizaje musical. No se trata sólo de disfrutar con el resultado sonoro, o de conjugar éste con unas sugerentes imágenes que nos aproximan a una obra de arte multifacética protagonizada por los miembros de una clase. En la actividad, el sonido actúa como envolvente de experiencias de respeto, hacia la música y las producciones sonoras propias y de los demás, y las interacciones que se producen entre los músicos y el profesor que los guía. La atenta actitud que esta tarea requiere, se transfiere a otros terrenos extra-musicales fuera del aula, así como el progreso en el aprendizaje de saber escuchar, imprescindible en el entorno escolar, pero también en la vida. La musicalización de los cuadros ha favorecido que el alumnado haya dotado de significado sus propias creaciones musicales junto a la visión global e integradora de la creación artística contemporánea a la que se han acercado con notable afinidad 
desde la creación propia. De esta forma, han construido experiencias musicales significativas a partir de sus vivencias musicales en el aula, asumiendo un papel activo en su propio aprendizaje como intérpretes y creadores, con una elevada motivación.

En definitiva, se refleja la conexión de una variedad de elementos que han interactuado desde una perspectiva holística en los procesos de aprendizaje. Estos han determinado la variedad de aprendizajes generados en el aula en el marco de los procesos creativos y participativos. Nos referimos a la motivación, las emociones positivas, el ambiente y bienestar, y la voz del alumnado, la identidad del grupo enmarcada en el desarrollo del proyecto didáctico.

Durante todo el proceso, el alumnado ha estado envuelto en su propio sonido, junto a los trazos, matices y tonalidades de los cuadros. Como consecuencia, la apertura sensorial que impulsa esta experiencia se proyecta y transciende más allá de la misma, porque ha convertido a los músicos de Secundaria en creadores de algo común e integrador, que se ha gestado a lo largo de todo el proyecto. Son sus palabras, las que expresan nuestros mejores argumentos:

"Ahora cuando veo un cuadro, escucho música" (Entrevista, 15 de junio de 2011, Alumna 8)

\section{Referencias biblográficas}

Acaso, M. (2013). Reduvolution. Madrid: Paidós Ibérica.

Alonso, C. (2008). Improvisación libre: La composición en movimiento. Baiona: Dos Acordes.

Alonso, C. (2014). Enseñanza y aprendizaje de la improvisación libre: Propuestas y reflexiones. Madrid: Alpuerto.

Batista, A. (2008). Arte flamenco: toque, cante y baile. Madrid: Alpuerto.

Berliner, P. (2002). Thinking in jazz: The infinite art of improvisation. Chicago, IL: University of Chicago Press.

Brinner, B. (2007). Music in central Java: Experiencing music, expressing culture. New York: Oxford University Press.

Burnard, P. (2000a). Making a Piece You Don't Play and Forget: Children Composing and the Role of Context. Australian Journal of Music Education, 1, 30-39.

Burnard, P. (2000b). Examining experiential differences between improvisation and composition in children's music-making. British Journal of Music Education, 17(3), 227245.

Burnard, P. (2007). Routes to understanding musical creativity. In L. Bresler (Ed.), International Handbook of Research in Arts Education (pp. 1197-1212). Dordrecht: Springer.

Burnard, P. (2012a). Musical Creativities in Practice. Oxford: Oxford University Press.

Burnard, P. (2012b). Rethinking musical creativity. In O. Odena (Ed.), Musical creativity: Insights from music education research (pp. 5-28). Aldershot, Hants: Ashgate.

Burnard, P. y Murphy, R. (2013). Teaching Music Creatively. London: Routledge.

Burnard, P. and Dragovic, T. (2014) Characterising communal creativity in instrumental group learning. Departures in Critical Qualitative Research, 3 (3), 336-362. 
Burnard, P., Apelgren, B. y Cabaroglu, N. (Eds.). (2015). Transformative teacher research: Critical issues in the future of learning and teaching. Amsterdam: SensePublishers.

Cage, J. (1961). Silence: lectures and writings. Middletown, Connecticut: Wesleyan University Press

Chóliz, M. y Gómez, C. (2002). Emociones sociales II (enamoramiento, celos, envidia y empatía). En F. Palmero, E. G. Fernández-Abascal, F. Martínez y M. Chóliz (eds.), Psicología de la Motivación y Emoción (pp. 395-418). Madrid: McGrawHill.

Cochran-Smith, M. y Litle, S. L. (2009). Inquiry as Stance: Practitioner Research for the Next Generation. New York: Teachers College Press.

Csikszentmihalyi, M. (1998). Creatividad: el fluir y la psicología del descubrimiento y la invención. Barcelona: Paidós.

Custodero, L. A. (2007). Origins and expertise in the musical improvisations of adults. British Journal of Music Education, 24(1), 77-98.

Dennis, B. (1991). Proyectos sonoros, Buenos Aires, Ricordi.

DeNora, T. (2000). Music in everyday life. Cambridge: Cambridge University Press.

Elliott, D. J. y Silverman, M. (2015). Music Maters: A new philosophy of music education ( $2^{\text {nd }}$ Ed.). New York: Oxford University Press.

Espinosa, S. (2005). La creación sonora en tiempo real. Una propuesta colectiva para la escuela secundaria. Aula de innovación educativa, 145, 28-33.

Fautley, M. y Savage, J. (2011). Cross-curricular teaching and learning in the secondary school The arts Drama, Visual Art and Design. Avingdom: Routledge.

Fautley, M. (2014). Listen, imagine, compose. Research Report. United Kingdom: Birmingham City University. Recuperado de http://resources.bcmg.org.uk/assets/ Resource/Downloads/Files/LIC-Final-Report-web-version.pdf

Frith, S. (2012). Creativity as a social Fact. In D. J. Hargreves, R. Macdonald y D. Miell (Eds.), Musical imaginations: multidisciplinary perspectives on creativity, performance and perception (pp. 62-72). New York: Oxford University Press.

Gardner, H. (2011). Inteligencias múltiples: la teoría en la práctica. Barcelona: Ed. Paidós. Geertz, C. (1988). La interpretación de las culturas, Barcelona, Gedisa.

Giglio, M. (2012). Cuando la colaboración creativa cambia la forma de enseñar. Santander: Ediciones Universidad Cantabria.

Gordon, J. (1996). Comportamiento organizacional. México, Prentice- Hall.

Green, L. (1988). Music on the Deaf Ears: Musical Meaning, Ideology and Education. Manchester, Manchester University Press.

Hargreaves, A. y Fullam, M. (2014). Capital profesional. Madrid: Morata.

Heron, J. y Reason, P. (1997). A participatory inquiry paradigm. Qualitative Inquiry, 3, $274-$ 294.

Hickey, M. (2003). Creative thinking in the context of music composition. In M. Hickey (Ed.), How and why to teach music composition: New horizons for music education (pp. 31-53). Reston, VA: MENC.

Hickey, M. (2009). ¿Can improvisation be "taught"?: A call for free improvisation in our schools. International Journal of Music Education, 27, 285-299. doi: 10.1177/0255761409345442

Hickey, M. (2015). Learning From the Experts. A Study of Free-Improvisation Pedagogues in University Settings. Journal of Research in Music Education, 62, 425-445. doi: $10.1177 / 0022429414556319$

INJUVE (2012). Informe. Juventud en España. Madrid: Gobierno de España. 
Jorquera, M. C. (2010). Modelos Didácticos en la Enseñanza Musical: el Caso de la Escuela Española. Revista musical chilena (Impresa), LXIV, 214, 52-74.

Koutsoupidou, T. y Hargreaves, D. J. (2009). An experimental study of the effects of improvisation on the development of children's creative thinking in music. Psychology of Music, 37(3), 251. doi: 10.1177/0305735608097246.

Kvale, S. (1996). InterViews: An Introducation to Qualitative Research Interviewing. London: SAGE.

Lage, C. y Cremades, R. (2017). The creation of music inspired by musical aids: A collaborative Action Research study of students motivation in music lessons. In P. Burnard, V. Ross, H. J. Minors, K. Powell, T. Dragovic, y E. Mackinlay (Eds.), Building Intercultural and Interdisciplinary Bridges: Where Practice Meets Research and Theory (pp. 85-94). Cambridge, UK: BIBACC. Recuperado de https://drive.google.com/file/ d/0BzB-0KN7nKv7Rk01eHNWZUgtQkU/view

Leung, B. W. (2004). A framework for undertaking creative music-making activities in Hong Kong secondary schools. Research Studies in Music Education, 23(1), 59-75. doi: $10.1177 / 1321103$ X040230010901

Ley Orgánica 1/1990, de 3 de octubre, de Ordenación General del Sistema Educativo (BOE $\mathrm{n}^{\mathrm{o}} .238$ de 04 de octubre de 1990).

MacDonald, R. (2014). We are all musical: Investigating improvisation as collaborative creativity. In M. S. Barrett (Ed.), Collaborative creative thought and practice in music (pp. 3-14). Farnham, Surrey, United Kingdom: Ashgate.

MacDonald, R., Hargreaves, D. J. y Miell, D. (Eds.). (2017). Handbook of musical identities. Oxford, UK: Oxford University Press.

Major, A. (2007). Talking about composing in secondary school music lessons. British Journal of music education, 24(2), 165-178. doi: 10.1017/S0265051707007437

Martínez, J.B. (1998). La voz del alumnado. Ausencia temporal de ciudadanía. Cuadernos de Pedagogía, 275, 56-65.

Matthews, W. (2012). Improvisando: La libre creación musical. Madrid: Turner Música.

McNiff, J. y Whitehead, J. (2002). Action Research: Principles and Practice (2 ${ }^{\mathrm{a}}$ Ed.). London: Routledge.

Miell, D. y Littleton, K. (Eds.). (2004). Collaborative creativity: Contemporary perspectives. London: Free Association Books.

Nooshin, L. y Widdess, R. (2006). Improvisation in Iranian and Indian Music. Journal of the Indian Musicological Society 36/37, 104-119.

Odena, O. (2014). Towards pedagogies of creative collaboration: Guiding secondary school students' music compositions. In Barrett, M. S. (Ed.), Collaborative Creative Thought and Practice in Music. Series: SEMPRE studies in the psychology of music (pp. 239-251). Farnham, UK: Ashgate Publishing.

Odena, O. (Ed.). (2012). Musical Creativity: Insights from Music Education Research. Farnham, UK: Ashgate Publishing.

Paynter, J. y Alston, P. (1970). Sound and Silence. Cambridge: Cambridge University Press. Peñalver, J. M. (2005). La aplicación didáctica de la improvisación musical y su didáctica en la enseñanza de la música en la Educación Obligatoria (Tesis Doctoral, Universitat de Valencia, Valencia). Recuperado de http://repositori.uji.es/xmlui/bitstream/10234/53119/1/44887.pdf Prevost, E. (1995). No sound is innocent. London: Copula.

Rice, P. y Ezzy, D. (1999). Qualitative Research Methods: A Health Focus. Melbourne: Oxford University Press. 
Saunders, L. y Somekh, B. (2009). Action Research and Educational Change: teachers as innovators. In S. Noffke y B. Somekh (Ed.), The Handbook of Educational Action Research (pp. 190-201). London: Sage Publications.

Sawyer, R. K. (2003). Group creativity: Music, theater, collaboration. Mahwah, NJ: Erlbaum.

Sawyer, R. K. (2008). Learning music from collaboration. International Journal of Education, 47, 50-59. doi:10.1016/j.ijer.2007.11.004

Schafer, M. (1965). El compositor en el aula. Buenos Aires: Ricordi.

Seddon, F. (2004). Empathetic creativity: The product of empathetic attunement. In D. Miell y K. Littleton (Eds.), Collaborative creativity. Contemporary perspectives (pp. 65-78). London: Fabooks.

Seddon, F. A. (2012). Empathetic creativity in music making. In O. Odena (Ed.), Musical creativity: insights from music education (pp. 133-147). Farnham, UK: Ashgate Publishing.

Self, G. (1991). Nuevos sonidos en clase: una aproximación práctica para la comprensión y ejecución de música contemporánea en las escuelas. Buenos Aires: Ricordi Americana.

Small, C. (2005). El acto de hacer música. En E. Roche y I. Domínguez-Palacios (Coords.), La dimensión humanística de la música: Reflexiones y modelos didácticos (pp. 73-93). Madrid: Ministerio de Educación, Cultura y Deporte. Secretaria General Técnica.

Soares, J. (2012). The nature of the engagement of Brazilian adolescents in composing activities. In O. Odena (Ed.), Musical Creativity: Insights from Music Education Research (pp. 113-132). Farnham, UK: Ashgate Publishing.

Thompson, W. (2006). Soundpainting: The art of live composition. Workbook I. New York: Walter Thompson.

Thompson, W (2009). Soundpainting, el arte de la composición en directo. Eufonía, 47, 7783.

Webster, P. (2002). Creative thinking in music: advancing a model. In T. Sullivan y L. Willingham (Eds.), Creativity and music education (pp. 16-33). Edmonton: Canadian Music Educator's Association.

Veloso, A.L y Carvahlo, S. (2012). Music composition as a way of learning: emotions and the situated self. En O. Odena (Ed), Musical Creativity: Insights from Music Education Research (pp. 73-92). Farnham, UK: Ashgate 